MATEC Web of Conferences 48,01004 (2016)

DOI: $10.1051 /$ matecconf $/ 20164801004$

(C) Owned by the authors, published by EDP Sciences, 2016

\title{
Analytical studies of torque motor tape active element
}

\author{
Antonina Dolgih ${ }^{1, a}$,Vladimir Martemyanov ${ }^{1}$ and Ivan Samodurov ${ }^{1}$ \\ ${ }^{1}$ National Research Tomsk Polytechnic University, 634050 Tomsk, Russia
}

\begin{abstract}
The paper presents analytical studies of the torque motor tape active element. The tape active element is a novel type of a motor's stator organization, where the conventional winding is replaced by a tape winding. Given the operation principle of proposed active element; its torque characteristics are then computationally found with using the finite element method (FEM). The results show the possibility of the optimal value of the relative electrode width, when the torque will be maximal. The analytical studies of the motor's torque over the number of tape winding coils allowed to receive the recommendations on choosing the number of coils.
\end{abstract}

\section{Introduction}

Recently, the significant attention is given to the development of the brushless torque motors novel types. The execution variant of the novel type torque motor is proposed in [1,2]. In the active element of this motor the conventional winding is replaced by a tape winding. The tape winding represents a spirally wound tape on a tubular base. The tubular base is a part of the motor case. The tape is made of electroconductive material and coated with a layer of insulation material. At both side edges of the tape there are narrow transverse cuts. The distance between cuts centers is equal to the pole division of the rotor magnetic system. If the ends of the wound tape connect to the DC source, the character of current flowing is determined by the transverse cuts. In the Figure 1 [1] it is shown that the current $J$ has two components: the longitudinal one $J_{l}$ directed along the tape and the transversal one $J_{t}$ directed across the tape towards coinciding with the rotation axis of the torque motor rotor. The poles of the rotor magnet are displayed by rectangles $\mathrm{N}$ and $\mathrm{S}$. The interaction of current transversal components $J_{t}$ with the magnetic field causes forces $F$, acting on the magnet. The total action of these forces causes the torque that tends to turn the magnetic system relative of the stationary tape winding. The longitudinal current components $J_{l}$ cause the forces $P$, acting from the side of magnet to the rotor suspension supports.

The benefits of the proposed active element include:

- Novel design and technological approach to the engine stator manufacture

- Great linear load due to the high current flowing through the tape winding and a good heat removal to the motor case

- Possibility of formation the required functional dependence of the motor's torque over the angular movement characteristic

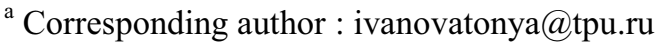




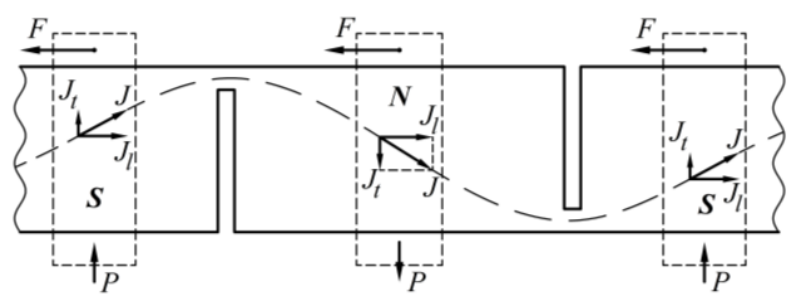

Figure 1. The active tape element fragment.

The operating principle of the proposed active elements is described in [2] by the example of the diagonally placed thin current-carrying conductor and magnetic field interaction. It is known that the diagonal character of the current flowing is also implemented in the windings of the coreless motors. Such windings are known as honeycomb, winding with skew-wound coils, zig-zag windings, Faulhaber winding [3-5].

The coils of the honeycomb winding are placed close or alternate to each other at an angle to the rotor element of cylinder. From design point of view the proposed variant of the active element is close to the approach implemented in the honeycomb winding. The main benefits of honeycomb windings include [5]:

- No cogging torque resulting in smooth positioning and speed control and higher overall efficiency than other DC motor types

- Extremely high torque and power in relation to motor size and weight

- Absolute linear relationship between load to speed, current to torque, and voltage to speed

- Very low rotor inertia which results in superior dynamic characteristics for starting and stopping

- Extremely low torque ripple and EMI

Torque motor with the tape active element has the dependence of motor's torque over the angular movement shown in the Figure 2, curve 1.

This dependence is obtained by calculation method [6] with using a numerical simulation of the current distribution through the tape winding in COMSOL Multiphysics. COMSOL Multiphysics software is a universal computer aid engineering software based on finite element analysis, which has a large set of functions for analyses and solution. For calculation of the dependence, the tape winding consists of 100 coils with 4 plates in each coil. Plate's length is $100 \mathrm{~mm}$, plate's width is $50 \mathrm{~mm}$ and plate's thickness is $0,1 \mathrm{~mm}$. Current is $50 \mathrm{~A}$, flux density in the air gap is $0,5 \mathrm{~T}$. Curve 2 in the Figure 2 represents the dependence of the motor's torque over the limited angular movement with the additional side cuts in the tape [6]. The additional side cuts allows to obtain the steady torque over the angular movement.

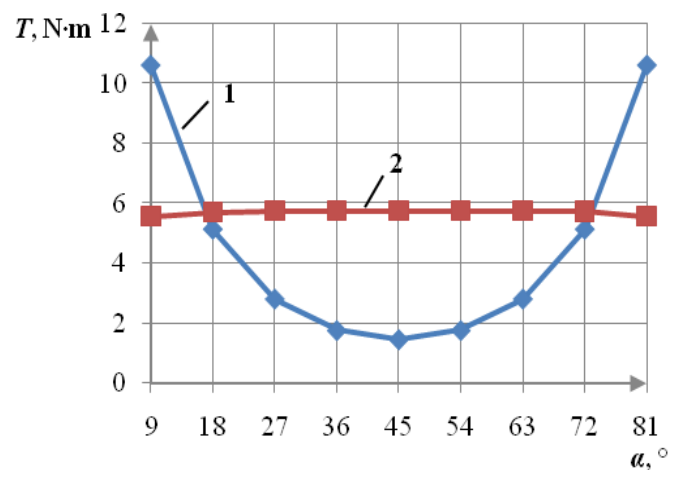

Figure 2. Calculation result of the motor's torque over angular movement.

The purpose of this work is to carry out the analytical studies of the tape active element single plate geometry influence and the number of tape winding coils on the motor's torque by the numerical simulation in Comsol Multiphysics. 


\section{Results and discussion}

As depicted in the Figure 1, the distributed direct current $J$ flows through the side electrodes of the single plate in the diagonal direction. Motor's magnetic system provides the uniform magnetic field with the constant flux density for some area of plate. The magnet center situated at some fixed point $x_{0}$ relative to the plate. Taking into account that the transverse current density component $j_{y}$ has the same value for the points with the same coordinates $\left(x_{i}, y_{i}\right)$, situated in all plate layers. The plate thickness $\Delta$ is constant. Suppose that the plate temperature, the applied voltage and the conductivity of the plate material are constant in time. In practice it is possible with the short time current flow, for example at the impulse mode.

In [6] it is shown that force generated by the interaction between the current-carrying plate and the permanent flux density has the following form

$$
F_{x}=B \cdot \Delta \cdot \int_{(x-c / 2)}^{(x+c / 2)} \int_{0}^{b} j_{y}(x, y) \cdot d x \cdot d y,
$$

where $B$ is a flux density in the air gap,

$c$ is a magnet width,

$b$ is a plate width.

The double integral in (1) represents the sum of the transverse current density components, flowing through the plate. Introduce the notation:

$$
D(x, y)=\int_{(x-c / 2)}^{(x+c / 2)} \int_{0}^{b} j_{y}(x, y) \cdot d x \cdot d y .
$$

If the value of $D(x, y)$ with some initial current $J=J_{0}$ is known, the double integral is denoted as $D\left(x, y, J_{0}\right)$. With current $J \neq J_{0}$ we have

$$
D(x, y, J)=\frac{J}{J_{0}} D\left(x, y, J_{0}\right) .
$$

In this case, (1) can be written in the form

$$
F_{x}=\frac{B \cdot \Delta}{J_{0}} \cdot J \cdot D\left(x, y, J_{0}\right) .
$$

The motor's torque is determined by the combined action of the tape winding single plates.

\subsection{The active element geometry influence to the motor's torque}

Consider the tape active element single plate geometry influence on the motor's torque. Firstly assess the force behavior over the changing of the voltage application electrode relative width $\sigma=\frac{b_{e}}{b}$, where $b_{e}$ is the electrode width (Figure 3). 


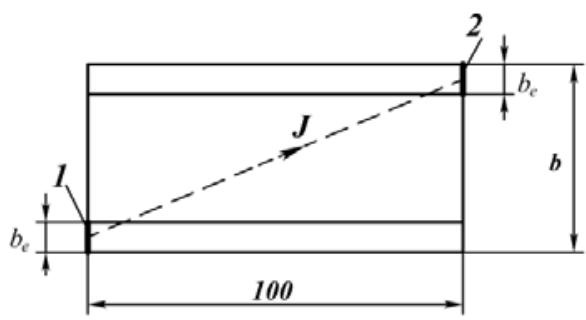

Figure 3. The single plate of the tape active element.

The operating current value $J$ at a constant voltage $U$, applied to the plate between the electrodes 1 and 2 depends on the relative electrode width $\sigma$. This occurs because the changing of the electrode cross-section area causes the change of the plate resistance and operating current.

The numerical experiment under specific values of $\sigma, c, x_{0}, J_{0}$ was carried out. In the Figure 4 the graph $J(\sigma)$ is represented. There is $\sigma$ varies from 0,1 to 0,5 with the constant voltage, in particular $U=0,01 \mathrm{~V}$.

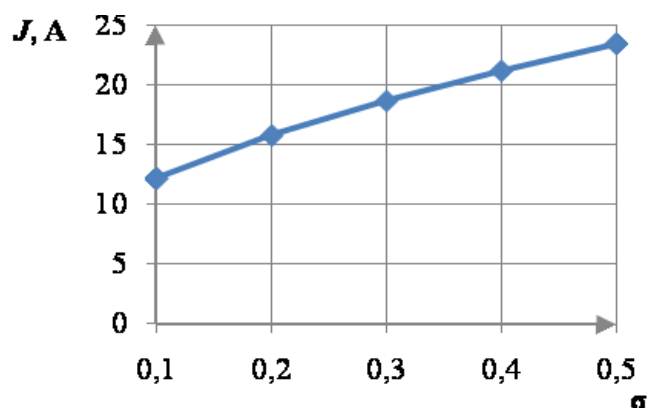

Figure 4. The graph of operating current over the relative electrode width with the constant voltage.

Note that the value $D\left(x, y, J_{0}\right)$ depends on the current streamlines steepness. The Figure 5 shows that increasing of $\sigma$ decreases the steepness.
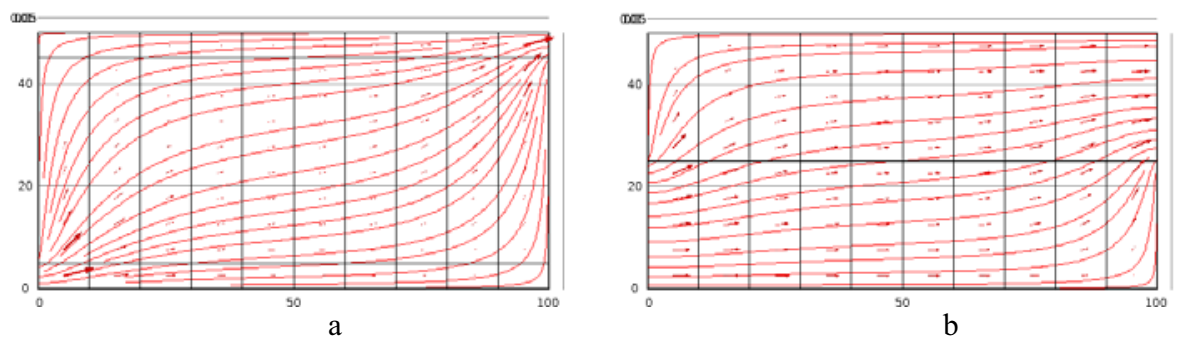

Figure 5. Current streamlines: a when $\sigma=0,1 ; \mathrm{b}$ when $\sigma=0,5$.

Define the value of $D\left(x, y, J_{0}, \sigma\right)$ with $\sigma$ from 0,1 to 0,5 (Figure 6). For providing the constant current $J_{0}$, flowing through the plate, in particular $1 \mathrm{~A}$, the applied voltage should be changed each time. The curve 1 shows the magnet location at the edge of the plate, curve 2 - at the center of the plate. 


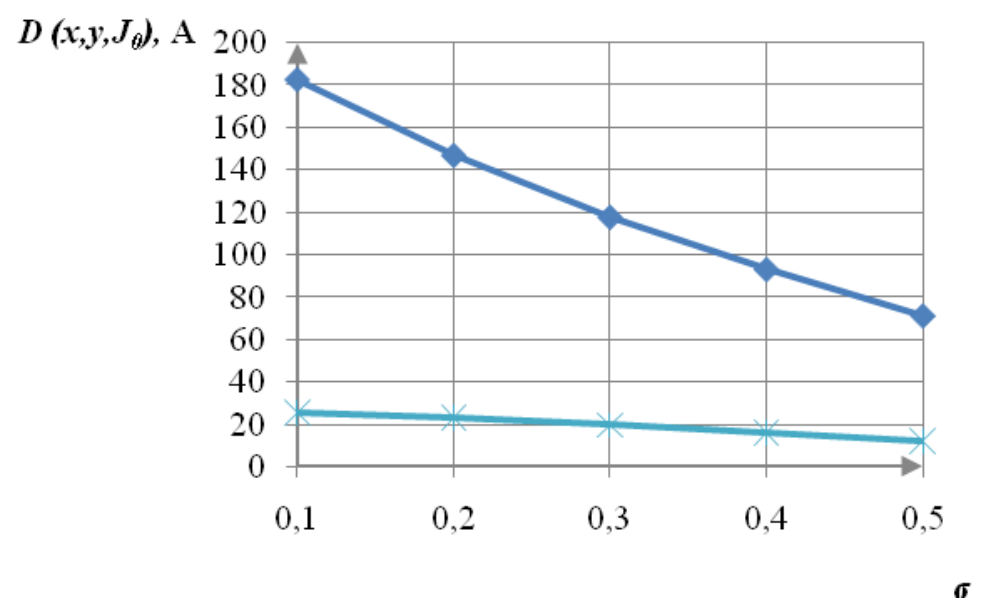

Figure 6. The double integral over the relative electrode width with the constant current $J_{0}$.

Thus the increasing of the $\sigma$ causes the increase in the current $J$ (Figure 4) and the decrease of the $D\left(x, y, J_{0}\right)$. Consequently, the simultaneous action of two opposite trends, affecting on the force value is observed. According that, (4) can be written as

$$
F_{x}\left(x_{0}\right)=\frac{B \cdot \Delta}{J_{0}} \cdot J(\sigma) \cdot D\left(x, y, J_{0}, \sigma\right) .
$$

The resulting force is the product of the above functions and has the form shown in Figure 7. Due to the fact that the both functions have a non-linear character, the expression for the force has an extremum at a certain value of the relative electrode width.

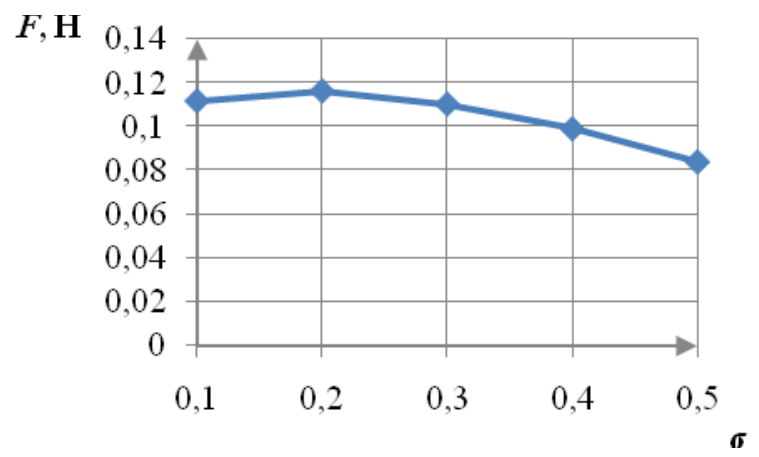

Figure 7. The resulting force over the relative electrode width.

\subsection{The motor's torque over the number of tape winding coils}

Consider how the motor's torque depends on the number of tape winding coils $N$. In this case, (4) takes the form

$$
F=B(\delta) \cdot \frac{\Delta}{J_{0}} \cdot \frac{U}{R \cdot N \cdot 2 p} \cdot D\left(x, y, J_{0}\right)_{x_{c}},
$$




\section{MATEC Web of Conferences}

where $B(\delta)$ is a flux density in the air gap; $\frac{U}{R \cdot N \cdot 2 p}$ is the tape winding operating current; $U=$ const - the voltage, applied to the tape; $R$ is the resistance of the single plate; $2 p$ is the number of plates in one coil; $D\left(x, y, J_{0}\right)_{x_{0}}$ is the double integral defined with the current $J_{0}$, and with the magnet center in the point $x_{0}$.

The force, created by the pack from $N$ one pole plates has the form

$$
F_{N}=B(\delta) \cdot \frac{\Delta}{J_{0}} \cdot \frac{U}{R \cdot N \cdot 2 p} \cdot N \cdot D\left(x, y, J_{0}\right)_{x_{0}} .
$$

The force, created by the all $2 p$ poles

$$
F_{N 2 p}=B(\delta) \cdot \frac{\Delta}{J_{0}} \cdot \frac{U}{R \cdot N \cdot 2 p} \cdot N \cdot 2 p \cdot D\left(x, y, J_{0}\right)_{x_{0}}=B(\delta) \cdot \frac{\Delta}{J_{0}} \cdot \frac{U}{R} \cdot D\left(x, y, J_{0}\right)_{x_{0}} .
$$

Then the motor's torque can be defined by the following way

$$
M=F_{N 2 p} \cdot r_{c p}=B(\delta) \cdot\left[\frac{r_{a v} \cdot \Delta}{J_{0}} \cdot \frac{U}{R} \cdot D\left(x, y, J_{0}\right)_{x_{0}}\right],
$$

where $r_{a v}$ is the average radius of the tape winding.

From (9) follows that the torque value depends only on flux density, and consequently from the value of the air gap. On the one hand the increased number of coils should increase the torque, but at the same time the current is reduced. Moreover, the larger number of coils will increase the air gap and decrease accordingly the flux density. Therefore, the number of coils must be minimal and defines by a permitted current. The permitted current $J_{p e r}$ must not damage the power supply and the tape winding

$$
\frac{U}{R \cdot N \cdot 2 p} \leq J_{p e r}, N \geq \frac{U}{2 p \cdot R \cdot J_{p e r}} .
$$

Provided that the magnetizing force of the permanent magnets is constant, we have

$$
B(\delta)=a \cdot \delta^{2}+b \cdot \delta+c=a \cdot\left(\delta^{2}+\frac{b}{a} \cdot \delta+\frac{c}{a}\right) .
$$

Assume the air gap value in the form of

$$
\delta=N \cdot \Delta+\Delta_{m}
$$

where $\Delta_{m}$ is the mechanical gap, including the thickness of the tubular base.

Substituting (11) and (12) into (9), we obtain

$$
M=k \cdot \Delta^{2} \cdot\left[N^{2}+B \cdot N+C\right]
$$


where $k=a \cdot\left[\frac{r_{a v} \cdot \Delta}{J_{0}} \cdot \frac{U}{R} \cdot D\left(x, y, J_{0}\right)_{x_{0}}\right], B=\frac{1}{\Delta}\left(2 \cdot \Delta_{m}+\frac{b}{a}\right), C=\frac{1}{\Delta^{2}} \cdot\left(\Delta_{m}^{2}+\frac{b}{a} \cdot \Delta_{m}+\frac{c}{a}\right)$.

Based on the possible application areas of the torque motors, among the basic requirements, applied to all the torque motors [7], it should be emphasized that the ratio of torque to the volume, weight, power consumption, management power should be higher to provide the quality of the motor. The power consumption defines by the following way

$$
P=J \cdot U=\frac{U^{2}}{2 p \cdot N \cdot R}
$$

Define the torque-power ratio

$$
\frac{M}{P}=\frac{k \cdot \Delta^{2}\left[N^{2}+B \cdot N+C\right]}{U^{2}} \cdot 2 p \cdot N \cdot R
$$

Substitute the coefficient $k$ into (8)

$$
\frac{M}{P}=\frac{a \cdot \Delta^{3} \cdot r_{a v} \cdot 2 p}{J_{0} \cdot U} \cdot D\left(x, y, J_{0}\right)_{x_{0}} \cdot N \cdot\left[N^{2}+B \cdot N+C\right]=K \cdot N \cdot\left[N^{2}+B \cdot N+C\right]
$$

After the simultaneous analysis of two expressions (13) and (16), taking into account (10), the number of coils $N$, which provide the characteristics of the motor defined by the customer determines.

\section{Conclusion}

The analytical studies of the tape active element single plate geometry influence on the motor's torque showed the possibility of the optimal value of the relative electrode width, when the torque will be maximal. The analytical studies of the motor's torque over the number of tape winding coils allowed to receive the recommendations on choosing the number of coils. A certain number of coils $\mathrm{N}$ will provide the motor characteristics defined by the customer.

\section{References}

1. V. Martemjanov, A. Dolgih (Ivanova), RF2441310 (2012)

2. V. Martemjanov, A. Dolgih (Ivanova), I. Siberian Conference on Control and Communications (SIBCON), 1 (2013)

3. R. D. Sedgewick, US4331896 A (1982)

4. J. F. Gieras, Permanent Magnet Motor Technology: Design and Applications (2010)

5. Dr. Fritz Faulhaber GmbH \& Co. KG, Technical information (2016)

6. A. Dolgih (Ivanova), V. Martemjanov, I. Plotnikova, Instruments and Systems: Monitoring, Control, and Diagnostics 4, 22 (2013)

7. L. Stolov, A. Afanasyev, Momentnye dvigateli postojannogo toka (1989) 